\title{
Effect of selenium supplementation on adiponectin concentration as a marker of type-2 diabetes risk in UK pregnant women
}

\author{
J. Mao ${ }^{1,2}$, S. C. Bath ${ }^{1}$, P. McCabe ${ }^{1}$, C. W. G. Redman ${ }^{3}$ and M. P. Rayman ${ }^{1}$ \\ ${ }^{1}$ Faculty of Health and Medical Sciences, University of Surrey, Guildford, GU2 7XH, ${ }^{2}$ The First Hospital of China \\ Medical University, Shenyang, China and ${ }^{3} J o h n$ Radcliffe Hospital, University of Oxford, Oxford, OX3 9DU
}

Though the effect of selenium (Se) on glucose metabolism is inconsistent, results of observational studies and randomized trials raised the suspicion that Se might increase the risk of type- 2 diabetes ${ }^{(1)}$. Adiponectin is a strong independent predictor of diabetes risk and higher adiponectin levels are associated with a lower risk of type-2 diabetes ${ }^{(2)}$. To test the effect of low-dose Se supplementation on plasma adiponectin concentration in pregnant women of relatively low Se status, we used stored plasma samples from the SPRINT (Se in Pregnancy Intervention) trial $^{(3)}$.

At 12 weeks of gestation, 230 pregnant women in the SPRINT trial were randomized to $60 \mu \mathrm{g}$ Se/day (high-Se yeast) or a yeast placebo for the duration of pregnancy. Two women with diabetes and one woman treated with thyroxine before conception were excluded. Whole-blood Se, plasma total adiponectin (by ELISA) and plasma glutathione peroxidase (GPx3) activity were determined at 12 and 35 weeks $^{(3)}$. Plasma selenoprotein P (SEPP1) was measured at 35 weeks ${ }^{(3)}$. As adiponectin concentrations at 12 and 35 weeks were highly correlated $(\mathrm{r}=0.764, \mathrm{P}<0.001)$, adiponectin at 12 weeks was incorporated in the general linear model (GLM) that compared adiponectin at 35 weeks in Se and placebo groups.

At baseline, there was no significant difference in adiponectin concentration between placebo and Se groups, even after adjusting for baseline BMI, whole-blood Se, age and gestational age at recruitment (Table). Adiponectin concentration significantly decreased during pregnancy in both placebo and Se groups $(\mathrm{P}<0.001)$ but did not differ between the groups, even in women in the bottom tertile of whole-blood Se at baseline $(\mathrm{P}=0.94)$. Though the percentage fall in adiponectin concentration was lower in the Se group than in the placebo group, the difference did not achieve significance $(P=0.59)$. Gestational diabetes in late pregnancy was diagnosed in two women in the placebo group but none in the Se group.

\begin{tabular}{|c|c|c|c|c|c|c|}
\hline \multirow[b]{2}{*}{ Gestational week } & \multicolumn{2}{|c|}{ Placebo group } & \multicolumn{2}{|c|}{ Se group } & \multirow[b]{2}{*}{ P-value ${ }^{b}$} & \multirow[b]{2}{*}{$\mathrm{P}$-value } \\
\hline & $\bar{n}$ & Adiponectin con. (mg/L) & $\bar{n}$ & Adiponectin con. (mg/L) & & \\
\hline 12 weeks & 112 & $11 \cdot 31(10 \cdot 36,12 \cdot 34)$ & 114 & $12 \cdot 23(11 \cdot 26,13 \cdot 27)$ & $0 \cdot 20$ & 0.09 \\
\hline 35 weeks & 108 & $8 \cdot 35(7 \cdot 65,9 \cdot 14)$ & 105 & $9.42(8 \cdot 54,10 \cdot 39)$ & $0 \cdot 08$ & $0 \cdot 23$ \\
\hline $\mathrm{P}$ value $^{\mathrm{a}}$ & & $<0.001$ & & $<0.001$ & & \\
\hline
\end{tabular}

Data are geometric medians (95\% confidence intervals). ${ }^{a} \mathrm{P}$ value from paired t-test, basline $v s 35$ wk; ${ }^{b} \mathrm{P}$ value, placebo group $v s$ Se group using a crude GLM; ${ }^{c} \mathrm{P}$

value, placebo group $v$ Se group, adjusted for baseline BMI, baseline whole-blood Se, age, gestational age at recruitment; baseline adiponectin was included in the GLM when comparing adiponectin at $35 \mathrm{wk}$

In the whole group, neither whole-blood Se nor GPx3 activity was correlated with adiponectin concentration at baseline $(\mathrm{r}=0.082$, $\mathrm{P}=0.22$ and $\mathrm{r}=0.104, \mathrm{P}=0.12$, respectively). At 35 weeks, the correlation between adiponectin and GPx3 activity almost reached significance in the $\operatorname{Se}$ group $(r=0.190, P=0.05)$ but not in the placebo group $(r=0.072, P=0.46)$. Results were similar for percentage change in adiponectin concentration from baseline to 35 weeks (Se group, $r=0.184, P=0.06$; placebo group, $r=0.082, P=0.40$ ).

In summary, in UK pregnant women of relatively low Se status, low-dose Se supplementation did not increase the risk of diabetes, as assessed by plasma adiponectin concentration, as previously found in a UK adult population ${ }^{(4)}$. The weak positive correlations between plasma adiponectin and GPx3 may suggest a potential benefit of Se supplementation in pregnancy on glucose metabolism which our study may not have been powered to detect.

SPRINT was funded by the Wellcome Trust; Dr. Mao was supported by a Nestlé Nutrition Research Fellowship and National Science Foundation of China (No. 81200571); Dr Bath was supported by an MRC Population Health Scientist Fellowship.

1. Rayman MP, Stranges S. (2013) Free Radic Biol Med 65, 1557-1564.

2. Li S, Shin HJ, Ding EL, et al. (2009) JAMA 302, 179-188.

3. Rayman MP, Searle E, Kelly L, et al. (2014) Br J Nutr 112, 99-111.

4. Rayman MP, Blundell-Pound G, Pastor-Barriuso et al. (2012) PLoS One 7, e45269. 